\title{
Sexual Biology and Reproduction in Crustaceans
}

\author{
by \\ Thanumalaya Subramoniam \\ Publisher: Academic Press \\ Print Book ISBN: 978-0-12-809337-5 \\ Pages: 526, Price: USD 127.50
}

The book on 'Sexual biology and reproduction in crustaceans' authored by Thanumalaya Subramoniam (TS) to be published by the Academic Press, an imprint of Elsevier, in 2017 is a comprehensive text on diverse topics in crustacean reproductive biology, long due and much sought as an independent book volume. The reading of the book instantly reveals the in-depth knowledge of the author in crustacean taxonomy, physiology and reproductive biology, and also his long experience and expertise in crustacean studies. As an author/editor of many previously published books, monographs, book chapters and reviews, the present title is a masterpiece work of TS. Given the vast literature on this economically and evolutionarily important group of aquatic/terrestrial invertebrates, the painstaking and time consuming efforts of the author to give an update of the subject are commendable and my deep appreciation and congratulations to TS on this very successful academic venture.

The book is organized into 14 chapters, glossary, references and subject index, extending over 508 pages. The salient features of the book include wellarticulated chapters with continuity in the flow of information, presentation of the chapters in a reviewstyle with fully-labeled hand-drawn and micrographic illustrations, information-rich tables, flow-charts, illustrated examples, highlighting biological features that are unique to crustaceans to develop suitable research models, and above all presenting ideas to stimulate further research.
The book provides an extensive coverage of sex determination, sex differentiation, sexual systems, mating systems, mating behavior, sex pheromones, reproductive cycle and environmental control of reproduction, oogenesis, endocrine regulation of vitellogenesis, yolk utilization and embryonic nutrition,

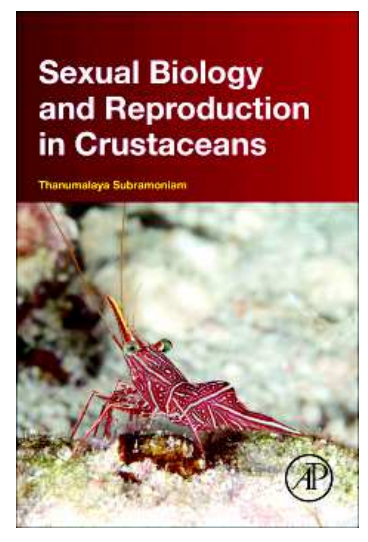
spermatogenesis, spermatophore and sperm transfer mechanisms, accessory reproductive glands and fertilization, covering the 14 chapters. These diverse topics will be of interest to biologists from all fields. The author has endeavored to project the common features shared by crustaceans and other groups of invertebrates and vertebrates and unique features that are exclusive to crustaceans.

The chapters on sex determination and sex differentiation (Chapters 1 and 2) illustrate that crustaceans practice diverse sex determining mechanisms ranging from the primitive polygenic system to the strong chromosomal sex determination with male or female heterogamety, epigenetic (environmental) factors (photoperiod, temperature, nutrition, hormones, and intracytoplasmic microbes like proteobacteria, protozoan symbionts that are transmitted along with germ cells in isopods and amphipods), and cytoplasmic sex factors ( $F$ and $\mathrm{f}$ ) 
that are exclusive to the group with the exception of aphids. These varied sex determining mechanisms are responsible for the distortion of sex ratios in the population and the prevalence of intersexuality, diversified sexual and mating systems (Chapter 3 and 4) and mating behaviors (Chapter 5). Crustaceans practice gonochorism, hermaphroditism (simultaneous and sequential), gynandromorphism (sexual mosaics) and parthenogenesis, and through illustrated models, the evolutionary transition between gonochorism and hermaphroditism through mixed sexual modalities is explained.

Crustaceans have developed effective communication channels through the production of sex pheromones (Chapter 6) to attract mates for successful mating. Of much interest to comparative biologists is the fact that uridine diphosphate (UDP), a product from UDP- $N$-acetyl glucosamine in chitin production (used in molting), acts as a urinary female pheromone attracting males for copulation in a wide range of crabs. The sex pheromone production in this manner brings in molting, female receptivity and copulation a sequential process.

Although crustaceans, depending on the latitude and depth of water in which they live, are continuous, semiannual and annual breeders, their reproductive cycle is influenced by the molting cycle (Chapter 7) so that there is a synergism between the reproductive and molting processes. Yolk synthesis and deposition in developing oocytes are central to oogenesis in ovaiparous animals such as crustaceans, insects and oviparous vertebrates. The biogenesis, uptake and deposition of yolk, processing and control mechanisms have a remarkable commonness among the egg-laying animals, which is reinforced by the phylogentic relationships of the vitellogenins. Crustacean vitellogenins and lipovitellins, yolk processing, and their regulation are extensively covered (Chapters 8-10). The presence of carotenoids being directly linked to the protein chains or esterified to the fattyacids of the lipovitellins is unique to crustaceans. Crustaceans maintain a distinct endocrine control of vitellogenesis, different from insects and other oviparous animals. These aspects are comprehensively reviewed in Chapter 9. There is a multitude of hormonal interplay in the control of vitellogenesis such as vitelloenesisinhibiting hormone, molt-inhibiting hormone, androgenic gland hormone in hermaphrodites, gonadstimulating hormone, methyl farnesoate, biogenic amines (serotonin and dopamine), and opioids. This list of the regulators also includes the potential roles of vertebrate GnRH-like molecules in the central nervous system and ovary, vertebrate gonadotropins, FSH, LH and hCG, in oogenesis and vertebrate type steroids like estradiol-17 $\beta$ and progesterone in vitellogenesis and meiotic maturation, respectively. The schematic diagram comparing the role of vertebrate type hormones in teleosts and crustaceans (Fig. 9-11, page 268) is a good working model for comparative research in these major groups.

Male reproductive strategies are as complex and diverse as female strategies. Crustaceans exhibit a great diversity in the testis and spermatozoon organization, specialized sperm transfer mechanisms (Chapter 12), and in the morphology, location and functions of the accessory sex glands in males and females (Chapter 13). The mode of fertilization of the gametes is much diverse and both external and internal occur in crustaceans. The process draws parallels with well studied sea urchin, amphibian and mammalian models. The mechanism of egg activation, sperm activation, egg-sperm interaction, acrosome reaction and cortical reaction show similarities with other well known models.

The bibliography is exhaustive and updated with recent literature, including citations from the author's own work and that of other Indian workers. Some minor formatting errors in the compilation could have been avoided and the author may recheck the bibliography before the final print of the book is released.

This compendium would serve as an authoritative reference book for crustacean reproductive biologists, developmental biologists, comparative endocrinologists, invertebrate biologists, evolutionary biologists and aquaculture scientists and would be an asset to personal and institutional library collections.

K. P. Joy

INSA Senior Scientist Department of Biotechnology

Cochin University of Science and Technology Kochi 682 022, Kerala 